\title{
The Viviparous-1 gene and abscisic acid activate the $C 1$ regulatory gene for anthocyanin biosynthesis during seed maturation in maize
}

\author{
Tsukaho Hattori, ${ }^{1}$ Vimla Vasil, Leonard Rosenkrans, L. Curtis Hannah, Donald R. McCarty, ${ }^{2}$ \\ and Indra K. Vasil \\ Vegetable Crops Department, University of Florida, Gainesville, Florida 32611 USA
}

\begin{abstract}
The Viviparous-1 (Vp1) gene is required for expression of the C1 regulatory gene of the anthocyanin pathway in the developing maize seed. We show that VP1 overexpression and the hormone, abscisic acid (ABA), activate a reporter gene driven by the $C 1$ promoter in maize protoplasts. Cis-acting sequences essential for these responses were localized. Mutation of a conserved sequence in the C1 promoter abolishes both ABA regulation and VP1 trans-activation. An adjacent 5-bp deletion blocks ABA regulation but not VP1 trans-activation. The latter mutant reconstructs the promoter of $c 1-p$, an allele that is expressed during seed germination but not during seed maturation. We suggest that VP1 activates $C 1$ specifically during maturation by interacting with one or more ABA-regulated transcription factors.
\end{abstract}

[Key Words: Maize; Viviparous-1 gene; C1 regulatory gene; abscisic acid]

Received October 31, 1991; revised version accepted February 7, 1992.

Purple anthocyanin pigments accumulate in specific tissues of the maize seed / the scutellum of the embryo and the aleurone cell layer of the endosperm) during the maturation period of seed development. At least eight genes $[A 1, A 2, B z 1, B z 2, C 1, C 2, R 1$, and Viviparous-1 (Vp1)] required for pigmentation of the seed are identified by mutants (Coe and Neuffer 1977). The C1, R1, and Vp1 genes have regulatory functions. The $\mathrm{Cl}$ protein has homology to the DNA-binding domain of the myb protooncogene (Paz-Ares et al. 1987) and contains an acidic transcriptional activation function (Goff et al. 1991). The $\mathrm{R} 1$ product is a helix-loop-helix protein with homology to myc (Ludwig et al. 1989). Recent functional analyses suggest that $\mathrm{Cl}$ and $\mathrm{R} 1$ interact to activate transcription of structural genes in the anthocyanin pathway (Goff et al. 1990; Roth et al. 1991). Whereas mutations in C1 and $R 1$ affect only anthocyanin synthesis, the anthocyaninless phenotype of the $v p 1$ mutant is associated with a general failure of seed maturation resulting in viviparous development of the embryo (Robertson 1955) and pleiotropic enzyme deficiencies in the aleurone (Dooner 1985). The viviparous phenotype is correlated with a reduced sensitivity of the vp1 mutant embryo to the plant hormone, abscisic acid [(ABA) Robichaud et al. 1980; Robichaud and Sussex 1986, 1987]. ABA has been widely

\footnotetext{
${ }^{1}$ Present address: Center for Molecular Biology and Genetics, Mie University, Tsu 514, Japan.

${ }^{2}$ Corresponding author.
}

implicated as the key hormone regulating seed maturation (Skriver and Mundy 1990).

Certain mutant alleles of $v p 1$ (vp1-McWhirter, vp11695, vp1-c821708, and vp1-A1) prevent anthocyanin synthesis but produce normal, nonviviparous seed (McCarty et al. 1989a), suggesting that control of the anthocyanin pathway is at least partially separable from regulation of embryo maturation. The block in anthocyanin synthesis in the $v p 1-R$ mutant is associated with failure to express the $C 1$ gene in developing seed tissues (McCarty et al. 1989b). This epistatic interaction suggested that $V p 1$ and $C 1$ may be part of a regulatory gene hierarchy. The $v p 1$ block in anthocyanin synthesis is conditional (McCarty and Carson 1990). If viviparous mutant seeds are removed from the ear prior to desiccation and allowed to continue to germinate in the light, anthocyanins accumulate in scutellum and aleurone tissues after several days. Chen and Coe (1978) described an allele of $c 1$, called $c 1-p$, that has an analogous effect. The $c 1-p$ seed is colorless at maturity; however, if exposed to light during development, seed tissues accumulate anthocyanins upon germination. Chen and Coe (1978) also showed that pigments accumulate in germinating (viviparous| seed of a $c 1-p, v p 1$ double mutant. These observations imply that $V p 1$ is only required for $C 1$ expression during seed maturation and that light can independently activate $C 1$ and $c 1-p$ alleles during germination (McCarty and Carson 1990). The phenotype of $c 1-p$ suggests that this allele is uncoupled from developmental control 
by $V p 1$ during maturation but remains responsive to light during germination.

In this paper we address the role of the $V p 1$ gene in integrating control of the anthocyanin pathway into a broader program of seed maturation. We have shown recently (McCarty et al. 1991) that $V p 1$ encodes a novel protein with properties of a transcriptional activator and that in maize protoplasts, VPl can trans-activate the promoter of $E m$, a downstream maturation-associated gene identified in wheat (Marcotte et al. 1988). Here, we show that both VP1 overexpression and ABA activate transcription of a reporter gene driven by $C 1$ promoter in maize protoplasts. We present evidence that ABA regulation and VPI trans-activation require separable, as well as shared, cis-acting sequences in the $C 1$ promoter.

\section{Results}

The $\mathrm{Cl}$ function is limiting for anthocyanin expression in vpl mutant tissue

We reasoned that if $\mathrm{Vpl}$ controlled the anthocyanin pathway by regulating the $C 1$ or $R 1$ genes, then constitutive expression of one or both regulatory genes in $v p 1$ mutant cells should complement the anthocyanin deficiency. Goff et al. (1990) have shown that constitutive expression of $\mathrm{Cl}$ and $\mathrm{Bl}$ (Bl being a member of the $R 1$ gene family) from the viral cauliflower mosaic virus (CaMV) 35S promoter complements the $c 1$ and $r 1$ mutations, respectively, when introduced into aleurone by microprojectile bombardment. The aleurone of $v p 1-R$ mutant kernels that were otherwise homozygous for $C 1$, $R 1$, and all other dominant genes required for seed pigmentation was exposed by removing the pericarp. Figure 1 shows that bombardment of $v p 1-R$ aleurone tissue with $35 \mathrm{~S}-\mathrm{Cl}$ and $35 \mathrm{~S}-\mathrm{B} 1$ together or $35 \mathrm{~S}-\mathrm{Cl}$ alone produced many pigmented cells. Pigment was visible within $12 \mathrm{hr}$ after bombardment of 17 (not shown)- or 25-day postpollination aleurones. In contrast, 35S-B1 alone did not effectively complement the anthocyanin deficiency. No pigmented cells were obtained in 17-day-old materials (not shown). At 25 days, 35S-B1 bombardment produced a few pigmented cells. These cells developed pigment more slowly than $\mathrm{Cl}$ - or $\mathrm{Cl}+\mathrm{Bl}$-transformed cells, becoming visible only after a 36-hr incubation. Constitutive expression of $\mathrm{Cl}$ is apparently sufficient to activate the anthocyanin pathway in $v p 1$ tissue, implying that the endogenous $\mathrm{R} l$ function is either already active or can be activated by $\mathrm{Cl}$. This experiment suggests that $\mathrm{C} 1$, but not $\mathrm{R} 1$, expression limits anthocyanin synthesis in the $v p 1$ mutant.

\section{$A B A$ and VP1 activate the $\mathrm{C} 1$ promoter in maize suspension culture protoplasts}

To examine the interaction of VPl with the $C 1$ gene, a transient expression system was used to quantify $C 1$ promoter function in maize cells under conditions where VPl expression and ABA hormone levels could be controlled. The 35S-Sh-Vpl effector and C1-Sh-GUS reporter plasmids diagramed in Figure 2 were introduced
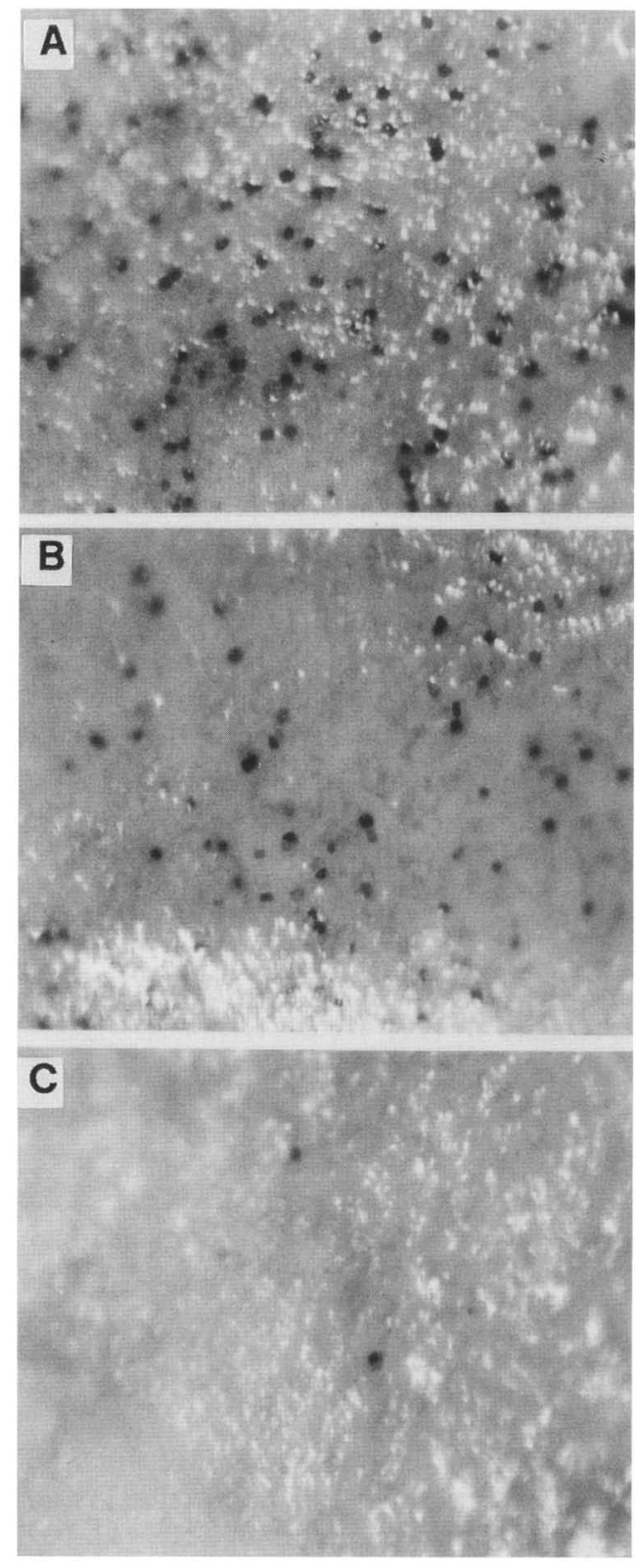

Figure 1. Constitutive overexpression of $\mathrm{C} 1$ complements anthocyanin synthesis in the vp1 mutant. CaMV $35 \mathrm{~S}-\mathrm{Cl}$ and CaMV 35S-B1 plasmids were introduced into $v p 1-R$ mutant aleurone cells (genotype W22 inbred, $v p 1-R, C 1, R 1$, and all other dominant genes required for aleurone pigmentation) exposed by removal of the pericarp from kernels 25 days after pollination by microprojectile-mediated transformation, as described by Klein et al. (1988) using a Dupont Biolistics gun. After bombardment, kernels were incubated for $36 \mathrm{hr}$ on agarose containing tissue culture medium (Murashige and Skoog 1962). 35S-GUS plasmid DNA $(1 \mu \mathrm{g} / \mu \mathrm{l})$ was included in each bombardment as a control for transformation. The aleurones shown in $A, B$, and $C$, respectively, were bombarded with $35 \mathrm{~S}-\mathrm{Cl}$ and $35 \mathrm{~S}-\mathrm{B} 1$ together $(1 \mu \mathrm{g} / \mu \mathrm{l}$ of each), 35S-Cl only, and $35 \mathrm{~S}-\mathrm{B} 1$ only. In the treatment shown in $C$, a total of three pigmented cells were detected on eight kernels bombarded with 35S-B1. Histochemical staining of representative kernels from each treatment for $\beta$-glucuronidase activity (Jefferson 1987) confirmed uniformity of transformation (not shown). 
by electroporation into protoplasts prepared from maize suspension culture cells. The $\mathrm{Cl}$-Sh-GUS reporter plasmid contained $609 \mathrm{bp}$ of 5 '-flanking sequence from the $C 1$ gene fused to the bacterial $\beta$-glucuronidase gene (vidA gene, GUS activity). In the $35 \mathrm{~S}-\mathrm{Sh}-\mathrm{Vpl}$ plasmid, expression of the full-length VP1-coding sequence is driven by the CaMV $35 \mathrm{~S}$ promoter. The first intron of the maize Sh1 gene was included in both gene constructs to enhance transient expression (Vasil et al. 1989). Cotransformation with $35 \mathrm{~S}-\mathrm{Sh}-\mathrm{Vpl}$ increased C1-Sh-GUS expression by about sevenfold, whereas cotransformation with a biologically neutral gene construct (35S-Sh-CAT) or a VPl antisense construct (35S-Sh-RVP) did not affect GUS activity significantly. Subsequent experiments (detailed below) showed that VPl trans-activation was dependent on specific sequences in the $C 1$ promoter, arguing strongly that the interaction measured was promoter specific. Addition of $10 \mu \mathrm{M}$ ABA to the culture medium after electroporation also activated C1-Sh-GUS strongly (11- to 14-fold). However, 35S-Sh-Vpl and ABA treatments in combination did not stimulate GUS expression above the level obtained with ABA alone. We addressed the possibility that endogenous ABA synthesis or expression of the endogenous $V p 1$ gene might affect C1-GUS activation. Subculturing the maize cells in media containing $10 \mu \mathrm{M}$ fluoridone, an inhibitor of ABA biosynthesis, prior to protoplast preparation, did not qualitatively alter ABA or VPl activation of C1-Sh-GUS /data not shown). Whereas VP1 could be detected in extracts of 35S-Sh-Vpl-transformed protoplasts, any endogenous expression in untransformed protoplasts was below the limit of detection by Western blotting (data not shown). In addition, ABA activation of $\mathrm{Cl}$-Sh-GUS was reduced $<20 \%$ by cotransformation of protoplasts with the $35 \mathrm{~S}$ Sh-RVP antisense plasmid.

To further explore the interaction of VPl and ABA in regulating $C 1$ promoter activity, we examined the $A B A$ dose response in the presence and absence of VP1 overexpression (Fig. 2C). ABA activation of Cl-Sh-GUS increased over a $0.1-100 \mu \mathrm{M}$ concentration range. This range is in good agreement with physiologically effective ABA concentrations for arresting maize embryo devel-
A

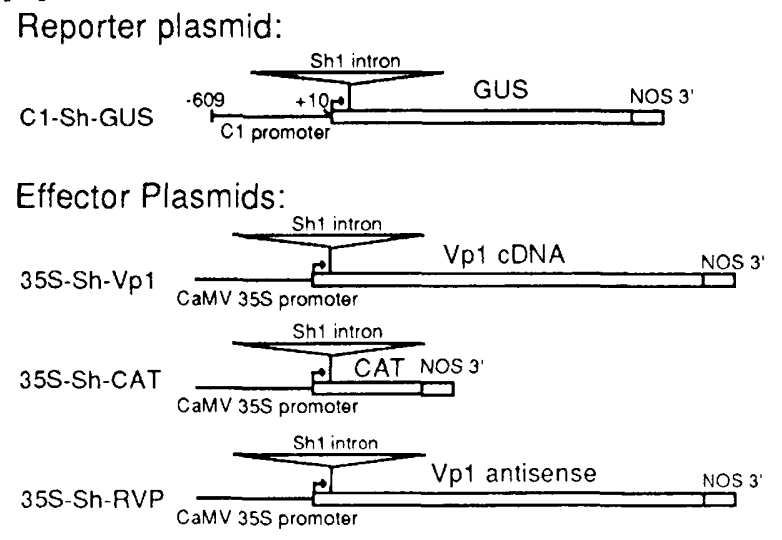

C

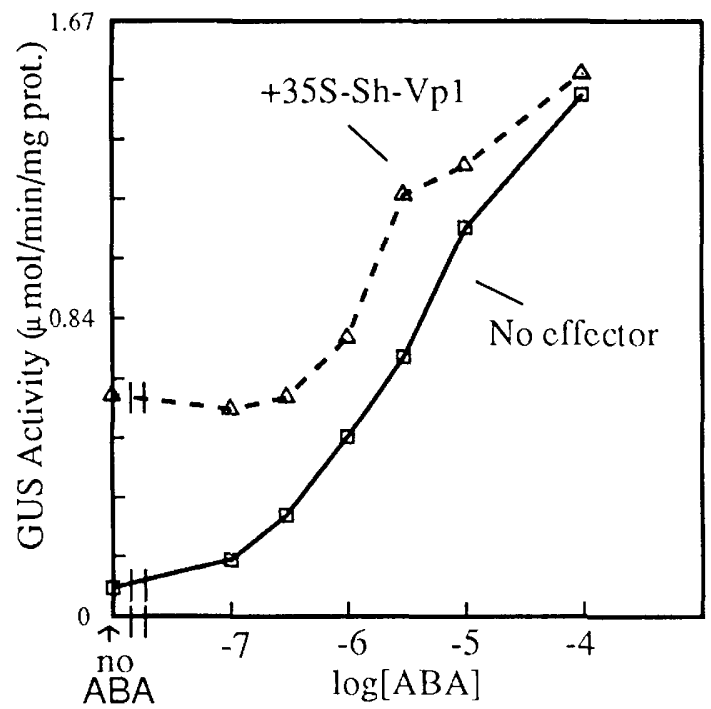

\begin{tabular}{|c|c|c|c|}
\hline \multirow{2}{*}{$\begin{array}{l}\text { Reporter } \\
\text { Plasmid }\end{array}$} & \multirow{2}{*}{$\begin{array}{l}\text { Effect or } \\
\text { Plasmid }\end{array}$} & \multicolumn{2}{|c|}{$\begin{array}{l}\text { GUS Activity } \\
\mu \mathrm{mol} \mathrm{MU} / \mathrm{min} / \mathrm{mg} \text { prot. }\end{array}$} \\
\hline & & $-A B A$ & $+A B A$ \\
\hline \multirow{3}{*}{ C1-Sh-GuS } & none & $0.24 \pm 0.04$ & $\begin{array}{c}2.75 \pm 0.3 \\
(11.4 x)\end{array}$ \\
\hline & $35 S-S h-V p 1$ & $\begin{array}{c}1.56+0.2 \\
(6.7 \overline{\mathrm{X}})\end{array}$ & $\begin{array}{c}2.82 \pm 0.2 \\
(117 x)\end{array}$ \\
\hline & 35S-Sh-CAT & $\begin{array}{c}0.26+0.01 \\
(1 .+\dot{x})\end{array}$ & $\begin{array}{c}3.43+0.3 \\
(14.3 \times \bar{x})\end{array}$ \\
\hline \multirow[t]{2}{*}{ C1-Sh-GUS } & none & $0.18 \pm 0.07$ & $\begin{array}{l}2.27 \pm 1.0 \\
(12.4 x)\end{array}$ \\
\hline & 35S-Sh-RVP & $\begin{array}{c}0.19+0.01 \\
(1.0 \bar{x})\end{array}$ & $\begin{array}{c}1.85 \pm 0.1 \\
(10.1 x)\end{array}$ \\
\hline
\end{tabular}

Figure 2. VPl overexpression and ABA activate a $C 1$ promoter-driven GUS gene in maize protoplasts. (A) Diagrams show the structures of the 35S-Sh-Vpl, 35S-Sh-CAT, 35S-Sh-RVP effector plasmids, and the $\mathrm{Cl}$-Sh-GUS reporter plasmid used for electroporation experiments. The effector genes were driven by the CaMV $35 \mathrm{~S}$ promoter with the first intron of the maize $S h 1$ gene included to enhance transient expression (Vasil et al. 1989). Construction of 35S-Sh-RVP and Cl-Sh-GUS is described in Materials and methods. 35S-Sh-Vpl (McCarty et al. 1991) and 35S-Sh-CAT /Vasil et al. 1989) have been described elsewhere. $(B)$ C1-Sh-GUS was introduced into maize cell suspension culture protoplasts by electroporation alone or together with an equal amount $120 \mu \mathrm{g}$ of DNA) of the indicated effector plasmid (see Materials and methods). After electroporation, each protoplast sample was split in two. Half was transferred into medium containing $10^{-4} \mathrm{M} \mathrm{ABA}$, and the remainder was cultured in medium containing no ABA. GUS activity was determined $40-45 \mathrm{hr}$ after electroporation. Values shown are means of three independent electroporations, with the S.E.M. indicated. Each effector was tested in a separate experiment. $\{C \mid$ Effect of $35 \mathrm{~S}-\mathrm{Sh}-\mathrm{Vpl}$ cotransformation on the ABA dose response of $\mathrm{Cl}$-Sh-GUS activation. Protoplasts were electroporated with C1-Sh-GUS alone ( $\square$ ) or Cl-Sh-GUS in combination with $35 S-S h-V p l(\triangle)$. Six electroporation reactions of each plasmid combination were pooled and then divided equally among media containing the indicated concentrations of ABA. Each point represents a single determination of GUS activity. Qualitatively similar results were obtained in five separate experiments. 
opment (Robichaud et al. 1980). The in vivo hormone concentration in endosperm most likely falls in the lower portion of this range (Neill et al. 1986; Jones and Brenner 1987). In cells cotransformed with $35 \mathrm{~S}-\mathrm{Sh}-\mathrm{Vp1}$, a substantial component of the total GUS response $(\sim 40 \%)$ was ABA independent. Consistent with the previous experiment, the interaction of VP1 and ABA was neither synergistic nor fully additive. 35S-Sh-Vpl cotransformation did not increase expression above the maximum level obtained with ABA alone. Similar results were obtained in five independent experiments (not shown), with the exception that in some protoplast preparations, the maximum ABA-induced GUS activity obtained in the 35S-Sh-Vpl cotransformed cells was actually lower than the maximum achieved in C1-GUStransformed cells treated with ABA alone. The basis for this apparent effect on the relative magnitude of the ABA-dependent response in some protoplast preparations is not known.

\section{Localization of $A B A$ and $\mathrm{Vpl}$ cis-responsive elements} in the $\mathrm{Cl}$ promoter

To further localize sequences in the $C 1$ promoter required for $\mathrm{ABA}$ and VP1 regulation, a series of $5^{\prime}$ dele- tions were made in the Cl-Sh-GUS gene and tested in the protoplast system (Fig. 3). Mutants deleted up to position - 157 relative to the transcription start remained qualitatively regulated by both ABA and VPl. Deletion of an additional 27 nucleotides to -130 abolished both $\mathrm{ABA}$ and VP1 activation. The -130 promoter, however, retained a significant basal activity.

Examination of the $C 1$ promoter revealed several sequences that are potentially conserved in other ABAregulated genes. Two sequences were detected (Fig. 4A) that resemble a putative $\mathrm{ABA}$ response element (ACGTGGC) identified in ABA-regulated genes of wheat (Guiltinan et al. 1989; Marcotte et al. 1989) and rice (Mundy et al. 1990). A perfect copy found in reverse orientation, at -97 , was within the minimally responsive promoter defined by the terminal deletions. A second motif with one mismatch occurs at -187 . To determine whether these sequences were critical for hormonal regulation, both sites were removed with small internal deletions. Although deletion of the element at -97 had a small quantitative effect on C1-Sh-GUS expression, neither mutant individually or in a double mutant combination substantially affected the $A B A$ response relative to basal expression (Fig. 4B). A second potential conserved sequence motif (designated the Sph element) was
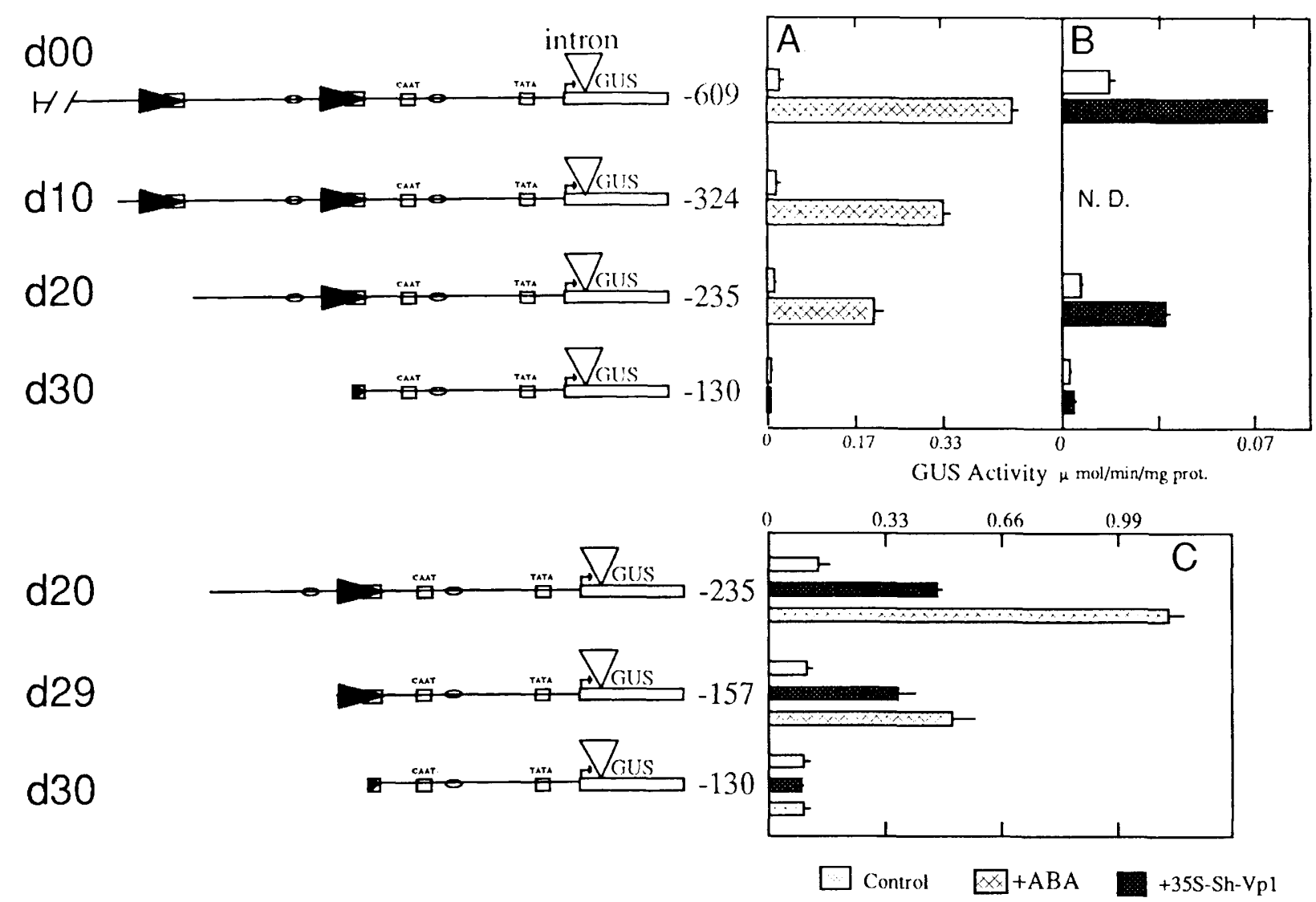

Figure 3. Localization of $C 1$ sequences required for ABA regulation and VP1 trans-activation in protoplasts. A series of 5 ' deletions were made in the promoter of Cl-Sh-GUS and tested for ABA and VPl regulation. (A) The relative ABA response of deletions up to $-130 .(B)$ The response of the same mutants to 35S-Sh-Vp1 cotransformation [(N.D.) not determined]. (C) The ABA and VP1 response of a deletion to -157 relative to the -235 and -130 deletion mutants. S.E.M. is indicated by lines extending from bars. 
A

$\begin{array}{lrl}\text { C1 (maize) } & -97 & \text { TACGTGGC } \\ & -187 & \text { TACGTTGC } \\ \text { RAB17 (maize) } & -205 & \text { GACGTGGC } \\ \text { RAB16A (rice) } & -181 & \text { TACGTGGC } \\ \text { EM (wheat) } & -235 & \text { CACGTGGC }\end{array}$

B

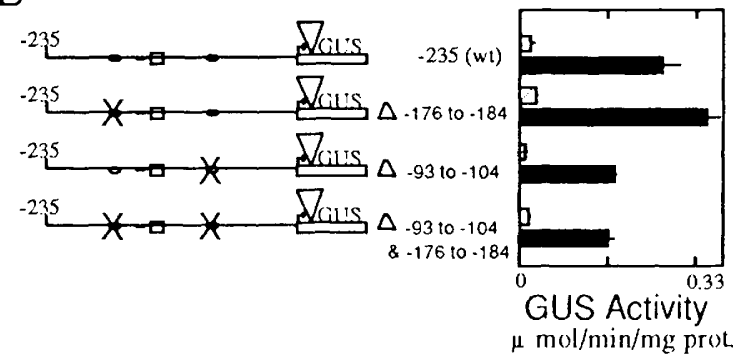

C

\begin{tabular}{lr|l|} 
C1 (maize) & -145 & TCCATGCATGCAC \\
& -258 & TGCATGCATGCAC \\
RAB17 (maize) & -111 & TCCACTCATGCAT \\
& -105 & CTCATGCATGCCE \\
RAB16A (Rice) & -79 & TCCACCCATGCCG \\
Em (wheat) & -341 & TIGCATGCATGCAA
\end{tabular}

Figure 4. Potentially conserved sequences identified in the $C 1$ promoter. (A) Comparison of two sequences in the $C 15^{\prime}$-flanking region that resemble Emla, a putative $\mathrm{ABA}$ response sequence identified by Marcotte et al. (1989), and similar sequences found in other ABA-regulated genes of maize (Vilardell et al. 1990) and rice (Mundy et al. 1990). The location of each sequence relative to the start of transcription is indicated. $|B|$ The Emla-like sequence motifs in C1 are not essential for ABA regulation. Small internal deletions were made in $\mathrm{Cl}-\mathrm{Sh}$ GUS(d20) that removed each of the Emla-like sequences described in $A$. Plasmids carrying single deletions, as well as both deletions, were tested for $\mathrm{ABA}$ activation in protoplasts. ( $U]$ ) $-\mathrm{ABA}_{i}(\square)+\mathrm{ABA}(10 \mu \mathrm{M}) .(C)$ Comparison of a repeat sequence present in $C 1$ promoter to similar sequences found in the same genes described in $A$. This motif was designated the Sph element because it frequently includes an SphI restriction site.

found in two copies in the C1 promoter (Fig. 4C). The more proximal copy overlapped the critical -157 to -130 region identified by the deletion analysis.

The -157 to -130 region was given further significance when it came to our attention that a sequence polymorphism within this interval distinguished wildtype $C 1$ alleles from the $c 1-p$ allele (B. Scheffler, P. Franken, E. Tapp, H. Seadler, and U. Wienand, pers. comm.). We had suggested previously that the $c 1-p$ phenotype was consistent with a regulatory mutation that would uncouple $C 1$ from developmental regulation by Vp1 (Mc-
Carty and Carson 1990). To confirm the sequence of the $c 1$-p promoter, a 400-bp genomic fragment was amplified by polymerase chain reaction (PCR), cloned, and sequenced (Fig. 5). A 5-bp direct repeat sequence found in wild-type $C 1$ alleles, at position -150 , is present in a single copy in $c 1-p$ (Fig. 5B). By comparison, within the $400-b p$ region, this was the only difference detected between $c 1-p$ and the wild-type C1 clone (Cone et al. 1986) used in this study.

A series of directed mutations were made to test sequences further in the -157 to -130 region (Fig. 6). The Sph sequence was disrupted by making a 6-bp deletion $(-136$ to -131$)$ in the $-235 \mathrm{C} 1-\mathrm{Sh}$-GUS construct (Fig. 6A). This mutation effectively abolished both ABA and VP1 activation. In addition, the sequence from - 151 to -147 was deleted to reconstruct the promoter sequence of $c 1-p$. This mutant was trans-activated by VP1, albeit less effectively than wild type. Importantly, however, it was not significantly activated by ABA. To define further the region essential for ABA and VP1 regulation, a series of multiple-base-change mutants that scanned farther in the 3 ' direction from -130 were tested (Fig. 6B). Although quantitative changes in basal and induced levels of expression were evident, these mutants remained qualitatively regulated by both ABA and VP1. To probe sequences still further 3 ', a deletion of sequences from -114 to -58 was tested (Fig. 6C). This mutant also responded to both ABA and VP1. In summary, only mutations within the $27-\mathrm{bp}-157$ to -130 region had a qualitative affect on ABA and VPl activation. The Sph and $c 1$ - $p$ deletion mutants, respectively, further resolved this

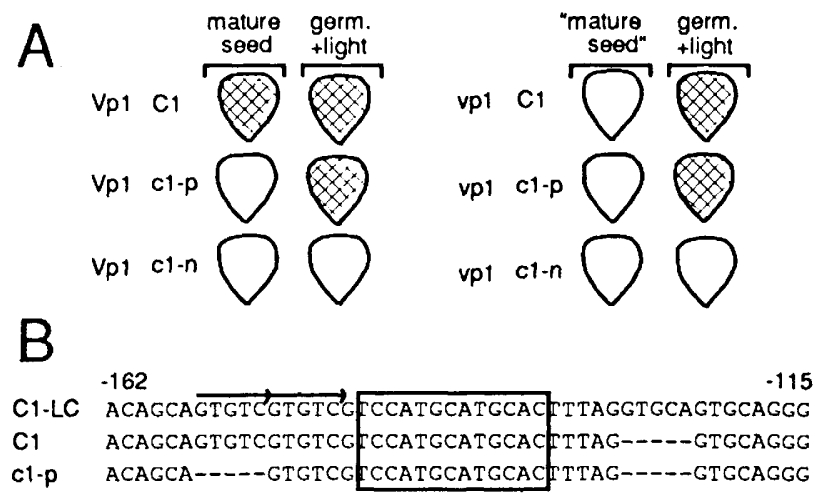

Figure 5. (A) Summary of $C 1, c 1$, and $c 1-p$ phenotypes. The $v p 1$ mutant does not prevent light-induced expression of $c 1-p$ /Chen and Coe 1978). Similarly, light exposure will induce anthocyanin accumulation in viviparous, $v p 1, C 1$ kernels. $(B)$ Sequence differences between $C 1$ wild-type alleles and $c 1-p$ in the proximal 5'-flanking region. A 400-bp genomic fragment of $c 1-p$ was amplified, cloned, and sequenced (see Materials and methods). The sequence comparison shows differences that distinguish the C1-LC (top line; Paz-Ares et al. 1987), C1 (middle line; Cone et al. 1986), and c1-p alleles (bottom line). The middle sequence was determined from the $C 1$ allele used to construct $\mathrm{Cl}$-Sh-GUS. No other differences were found in the sequenced region. Additional polymorphisms occur $3^{\prime}$ to the coding region in $c 1-p$ (Wienand et al. 1990). 

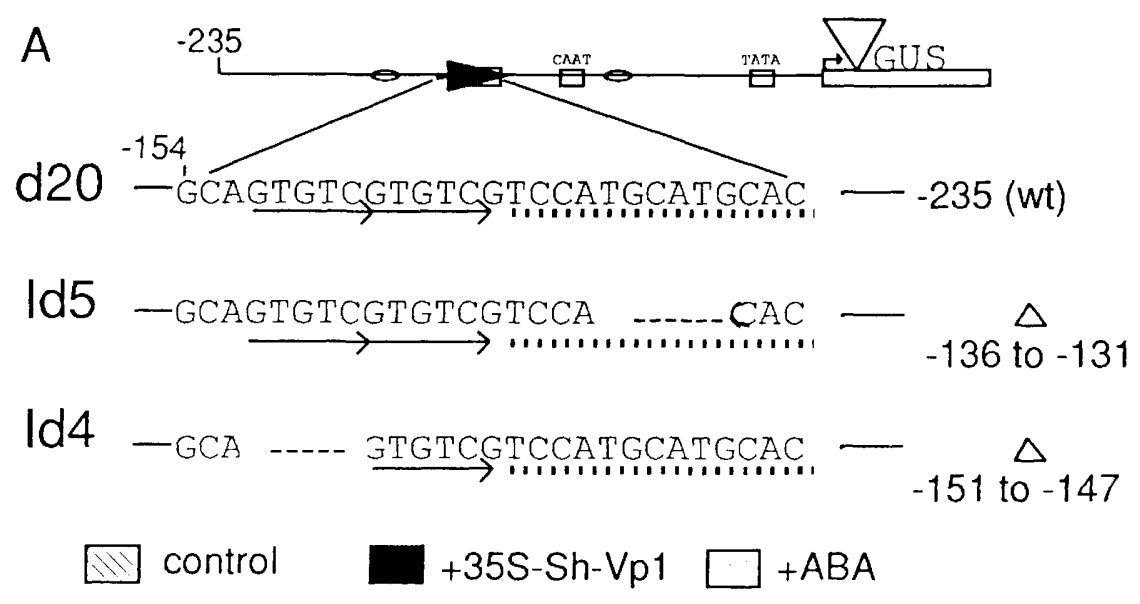

B

$$
\text { C }
$$$$
\text { B }
$$$$
\text { Mutant }
$$$$
\text { d20 } \overrightarrow{\text { GTGTCGTGTC }} \longrightarrow \overrightarrow{\text { TCCATGCATGCACFTTAGGTGCAGGGCCTCAACT }}
$$$$
8283
$$$$
8081
$$$$
7879
$$$$
7677
$$$$
\text { (- GCTC- }
$$

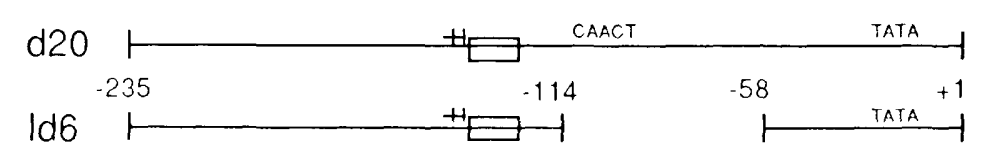

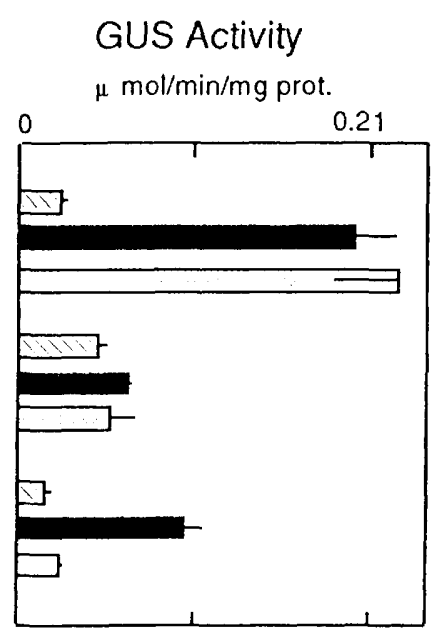

GUS Activity

pmol/min/mg prot.

\begin{tabular}{|c|c|c|}
\hline Control & $A B A$ & VP1 \\
\hline $20 \div 3$ & $433 \div 8$ & $125 \div 5$ \\
\hline $73 \div 5$ & $984 \div 42$ & $283 \div 8$ \\
\hline $32 \div 12$ & $500 \div 83$ & $250 \div 8$ \\
\hline $6.7 \pm 6.7$ & $267 \pm 8$ & $97 \div 17$ \\
\hline $6.7 \pm 3$ & $450 \div 83$ & $97 \div 17$ \\
\hline
\end{tabular}

Figure 6. Mutagenesis of sequences essential for ABA and VPl regulation of $C 1$ in protoplasts. (A) ABA regulation and VP1 transactivation of C1-Sh-GUS(d20) and two internally deleted derivatives were compared. In the Id5 mutant 6 bp was deleted in the Sph element. The Id $4|c 1-p|$ mutant contained a 5 -bp deletion that reconstructed the $c 1-p$ promoter sequence. GUS activities shown are means of three independent electroporation reactions. The S.E.M. is indicated by lines extending from bars. $(B)$ Multiple-base-change derivatives of $\mathrm{Cl}$-Sh-GUS(d20) as in $A$, except that each value is the mean of two electroporations. $(C)$ The response of Id6, an internal deletion of sequences between -114 and -58 , is compared with C1-Sh-GUS(d20). Means of three electroporations are shown.

region into a $3^{\prime}$ region required for both responses and an upstream region required specifically for $A B A$ activation.

\section{Discussion}

Our results indicate that VP1 controls the anthocyanin pathway in the developing maize seed primarily through regulation of the $C 1$ gene. Transient expression of $C 1$ is sufficient to complement pigment synthesis in $v p 1 \mathrm{mu}-$ tant tissue. This observation suggests that $C 1$ expression limits the anthocyanin pathway in the $v p 1-R$ mutant and that a direct interaction of VP1 with the downstream structural genes in the anthocyanin pathway is not essential. Expression of the $R 1$ regulatory gene, on the other hand, is not limiting in the $v p 1$ mutant. The $R 1$ apparently does not require VP1 for expression. The demonstration that overexpression of VPl in maize protoplasts activates transcription from the $C 1$ promoter supports further the idea that $V p 1$ and $C 1$ constitute a gene hierarchy (Fig. 7). Both $V p 1$ and $C 1$ encode transcription factors. The $\mathrm{Cl}$ product has homology to the DNA-binding domain of myb (Paz-Ares et al. 1987) and contains a functional transcriptional activation domain (Goff et al. 1991). C1 and a helix-loop-helix protein encoded by members of the $R 1$ gene family (Ludwig et al. 1989) apparently interact to activate transcription of structural genes of the anthocyanin pathway in seed tissues (Goff et al. 1990; Roth et al. 1991). Domain-switching studies have shown that VP1 also contains a potent transcriptional activation domain and that this function is required for trans-activation of a target gene in maize protoplasts (McCarty et al. 1991).

$V p 1$ has been implicated in ABA hormone responses of seed tissues (Robichaud et al. 1980; McCarty et al. 1991). Here, we have shown that activity of the $C 1$ promoter is also regulated by ABA. The correlation between the colorless, mature seed phenotype of the $c 1-p$ allele and the failure of the $c 1$-p-like promoter to respond to ABA in 


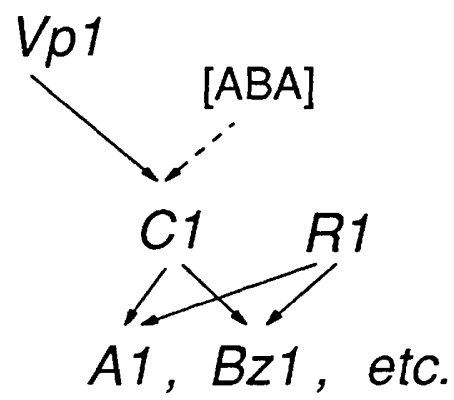

Figure 7. Proposed regulatory pathway controlling anthocyanin biosynthesis in the developing seed. Both $C 1$ and $R 1$ are required for expression of anthocyanin structural genes /Goff et al. 1990). VP1 and ABA regulate the $C 1$ gene during seed maturation.

protoplasts suggests strongly that $\mathrm{ABA}$ is important in regulating $C 1$ expression during seed development. Although $c 1-p$ also carries DNA rearrangements $3^{\prime}$ to the C1-coding sequence (Wienand et al. 1990; B. Scheffler, P. Franken, E. Tapp, H. Seadler, and U. Wienand, pers. comm.), which could concievably affect expression, we suggest that the 5-bp rearrangement in the promoter is sufficient to disrupt $A B A$ regulation and, as a result, uncouple the anthocyanin pathway from the maturation developmental pathway. Consequently, $c 1-p$ expression is delayed until after germination when, by default, it is activated by light (Chen and Coe 1978). In a vp1 mutant background, wild-type $C 1$ and $c 1-p$ alleles have apparently similar phenotypes |(delayed light-dependent pigmentation) Chen and Coe 1978; McCarty and Carson 1990], suggesting that VP1 is required for ABA activation of $C 1$ during seed development. Failure to respond to ABA would therefore apparently be the underlying basis for the pleiotropic effects of $v p 1$ on anthocyanin synthesis and maturation (Robichaud et al. 1980). In the protoplast system, VP1 contributes most strongly to $C 1$ activation at low hormone concentrations $\left(10^{-7}\right.$ to $10^{-5} \mathrm{M}$ ABA). This is consistent with evidence that ABA concentrations in developing maize endosperm are $<1 \mu \mathrm{M}[0.1-0.5$ nmole/g of fresh weight (Neill et al. 1986; Jones and Brenner 1987)]. At these low concentrations, VPl action may be essential to augment ABA activation of $C 1$.

Although overexpression of VP1 in protoplasts causes activation of $C 1$ and, to some extent, $c 1-p$ promoters in the absence of $\mathrm{ABA}$, it is unclear what role this hormoneindependent response has in normal development. VPI alone is apparently not sufficient to activate $c 1-p$ in situ. On the other hand, hormone-independent activation of the wild-type $C 1$ allele could explain the long-standing observation that ABA-deficient mutants of maize (e.g., $v p 5$ ) are generally not deficient in anthocyanins (Robertson 1955). One possibility is that the $c 1-p$ and $C 1$ promoters have different affinities for VPl and that saturating levels of VP1, not normally present in situ, partially mask this difference.

The fold activation of $C 1$ that we obtain in protoplasts with VP1 $(\sim 10$-fold at $1 \mu \mathrm{M} A B A)$ is modest compared with the level of activation ( $\sim 100$-fold) of anthocyanin structural genes that has been achieved by overexpression of the $\mathrm{Cl}$ and $\mathrm{Rl}$ proteins in maize tissues (Goff et al. 1990; Roth et al. 1991). Although it is possible that unknown factors limit full activation of $C 1$ in the protoplast system, the low steady-state level of $C 1 \mathrm{mRNA}$ detected in seed tissues (Paz-Ares et al. 1987; McCarty et al. $1989 \mathrm{~b}$ ) is consistent with a low rate of transcription of the $C 1$ gene in vivo. In any case, the degree of induction that we observe is probably sufficient to control the pathway given the potential for amplification downstream in the cascade afforded by the apparent sensitivity of the structural genes to activation by Cl (Goff et al. 1990, 1991; Roth et al. 1991). If, for example, we assume a simple dose response curve for $\mathrm{Cl}$ activation of the $\mathrm{Bz} 1$ gene that saturates over a 2 log-cycle range of protein concentration with a maximum activation of 100 -fold, then a 10-fold change in $\mathrm{Cl}$ expression could produce a 50 -fold change in $B z 1$ expression. Higher amplification is possible if cooperativity or other synergistic interactions play a role. Moreover, $\mathrm{Cl}$ protein may accumulate in aleurone cells over a period of time.

The origin of the $c 1-p$ allele has interesting implications for the evolution of the maturation pathway in maize. Because $c 1-p$ occurs as a natural variant in maize populations (Chen and Coe 1978), it is unknown whether the C1- or $c 1$-p-like promoter sequence is the ancestral form. If the $C 1$ form arose from a $c 1-p$-like progenitor through duplication of the GTGTC sequence, it may be that the anthocyanin pathway became integrated into the maturation pathway only recently in the evolution of maize. Polymorphisms involving small direct duplications, possibly created by transposable element visitation or other mechanisms, occur frequently among alleles of other maize genes (e.g., Zack et al. 1986). Evidence for the direction of this change in the $c 1$ locus may still exist in related grasses, such as Teosinte.

A relationship between ABA regulation and VPl is suggested further by the analysis of cis-regulatory sequences in the $C 1$ promoter. Essential sequences for both responses map to a 27 -bp region of the $C 1$ promoter $(-157$ to -130$)$. This sequence can be resolved further into a region specifically required for $A B A$ activation and a region required for both hormonal regulation and VP1 trans-activation. A 6-bp deletion in the potentially conserved Sph element abolishes qualitative regulation by $\mathrm{ABA}$ and VPl without reducing basal expression. Although it is possible that this deletion affects spacing between two cis-acting elements rather than disrupting a critical sequence, mutagenesis or deletion of sequences in the 72-bp region immediately downstream failed to detect other essential sequences. Sequence motifs resembling the Sph element occur in the promoter regions of several other ABA-regulated genes isolated from cereals (Mundy et al. 1990; Marcotte et al. 1989). We have shown recently that at least one of these genes is strongly trans-activated by VP1 in maize protoplasts (McCarty et al. 1991). An 8-bp CATGCATG motif, present in the core of the Sph element, is found frequently in $5^{\prime}$-flanking sequences of seed-specific plant genes but rarely in nonseed genes (Dickinson et al. 1988). 
This sequence is also like the similarly named Sph motifs of the SV40 virus early promoter (Zenke et al. 1987).

The ABA-specific region that includes at least part of the sequence from -157 to -147 bears little obvious similarity to the Emla element (ACGTGGC) implicated in ABA regulation of the $\mathrm{Em}$ gene of wheat (Marcotte et al. 1989). The Emla sequence is apparently essential for $\mathrm{ABA}$ regulation of $E m$ in rice protoplasts (Guiltinan et al. 1990). Two sequence motifs in the C1 promoter that resemble closely the Emla sequence are evidently not required for $\mathrm{ABA}$ regulation. Although both $E m$ and $C 1$ are trans-activated by VP1 (McCarty et al. 1991), different transcription factors may mediate $\mathrm{ABA}$ regulation of these genes. This possibility is underscored by strikingly different interactions between VPl trans-activation and the $\mathrm{ABA}$ response exhibited by the $\mathrm{Em}$ and $C 1$ promoters. VP1 and ABA interact synergistically in activating Em (McCarty et al. 1991), whereas for the C1-driven reporter gene construct the combined response to VP1 and $\mathrm{ABA}$ is less than additive. These features suggest that the interaction of VPl with these two genes may differ mechanistically. It is intriguing in this respect that several known $v p 1$ mutations prevent anthocyanin synthesis without blocking maturation (McCarty et al. 1989a; McCarty and Carson 1990). At least one of these mutants, vp1-McWhirter, produces a truncated protein (McCarty et al. 1989a, 1991; C.B. Carson and D.R. McCarty, unpubl.). The altered protein can apparently activate many functions associated with maturation but not $C 1$ and the anthocyanin pathway.

The partial separation of $\mathrm{ABA}$ and $\mathrm{VPl}$ regulatory functions suggests that VPI may not be an integral component of the ABA signal transduction pathway, although it is possible that the pathway is branched and that one arm includes VP1. It is reasonable to expect that several factors may bind in the region between -157 and -130 . We speculate that VP1 or a complex that includes VP1 may interact in this region. It is not known whether VP1 binds DNA. It lacks all known conserved DNAbinding motifs (McCarty et al. 1991). Moreover, VP1 expressed in Escherichia coli or by in vitro translation does not interact with oligonucleotide fragments containing the -157 to -130 region of $C 1$ in gel-shift experiments (T. Hattori and D.R. McCarty, unpubl.). VPl could interact with this region primarily through protein-protein contacts, as has been suggested for some other transcriptional activators (e.g., Triezenberg et al. 1988). A sequence that overlaps the conserved Sph element is apparently required for both $\mathrm{ABA}$ and VP1 regulation. $\mathrm{A}$ similar CATGCATG motif has been associated with seed-specific gene expression in plants (Dickinson et al. 1988). It seems unlikely that all genes in which this sequence has been identified are regulated by ABA or VP1. One possibility is that VP1 may interact with multiple transcription factors in a way that integrates the ABA signal transduction pathway with other intrinsic developmental signals, possibly specifying tissue or positional information. Failure to achieve this integration would be consistent with the tissue-specific, hormone-insensitive phenotype of $v p 1$.

\section{Materials and methods}

Transient expression in protoplasts

Protoplasts were prepared from an embryo-derived maize suspension cell line as described previously (Vasil et al. 1989). The cell line was derived from embryos of a DK XL80 hybrid (Dekalb Seed, Inc., Dekalb, IL). Most or all commerical hybrids are wildtype $V p 1$ and carry recessive, nonpigmenting alleles of $c 1$ and $r 1$. The genotype with respect to other pigment loci was not determined. Electroporation reactions included $4 \times 10^{6}$ cells and $20 \mu \mathrm{g}$ each of effector plasmid and reporter plasmid. Unless indicated otherwise, each treatment was replicated in triplicate. Protoplasts were cultured in growth media (Vasil et al. 1989) for $40-45 \mathrm{hr}$ after electroporation and then collected by centrifugation and lysed. Soluble extracts were assayed for glucuronidase (Jefferson 1987). GUS activities were normalized to extracted protein (Bradford 1976). The S.E.M. for GUS activities obtained from independent electroporations done using the same protoplast preparation was typically $<20 \%$ of the mean, indicating that variation in transformation efficiency within experiments was low. Because greater variation was observed among protoplast preparations made on different days, only treatment comparisons made within a single experiment were considered valid.

Cloning and sequence analysis of a cl-p promoter fragment

A fragment spanning the region from +16 to -390 of the $c 1-p$ gene was amplified by PCR from genomic DNA isolated from a $c 1-p$ stock (a gift of Ed Coc, University of Missouri, Columbia) size enriched for a $9.0-\mathrm{kbp} \mathrm{BamHI}$ fragment containing the gene by preparative agarose gel electrophoresis. Single-base changes were incorporated into the respective primers $\left(5^{\prime}\right.$-CCATCGAGCTCGCTCTCTCG $-3^{\prime}$ and $5^{\prime}$-CTGGGGATCCTTAGTTACTG-3') to incorporate Sstl and BamHI sites for convenient cloning into pUC19. This clone and a corresponding region of the wild-type $C 1$ allele used in the GUS constructs (Cone et al. 1986) were sequenced by dideoxy chain termination on a Dupont Genesis 2000 DNA Analysis System (E.I. du Pont de Nemours, Inc., Wilmington, DE).

\section{Plasmid constructions}

All nucleic acid manipulations were carried out using standard procedures (Maniatis et al. 1982). Restriction enzymes and DNA-modifying enzymes were used according to manufacturers' instructions. Synthetic oligonucleotides were prepared using an Applied Biosystems DNA synthesizer and purified by either anion exchange chromatography (Mono-Q, Pharmacia, Uppsala, Sweden) or polyacrylamide gel electrophoresis.

Construction of $35 \mathrm{~S}-\mathrm{Sh}-\mathrm{Vpl}$ is described in McCarty et al. (1991). To construct the VPl antisense plasmid, 35S-Sh1-RVP, the cVP23 cDNA clone (McCarty et al. 1991) was cloned into the EcoRI site of pSP72 (Promega) in the orientation that placed the $5^{\prime}$ end proximal to the SstI site of the vector. The cVP23 fragment was cut out with EcoRV and SstI and ligated to a backbone fragment prepared from pBI221 (Bevan 1984) by digestion with SmaI and SstI, which generated 35S-RVP. An XbaIPst I fragment containing the first intron of the Sh1 gene was prepared from 35SIfCN (Vasil et al. 1989) and subcloned into pBluescript $(-$ ) (Stratagene, Inc., La Jolla, CA). The fragment containing the intron was prepared by digestion of this plasmid with $\mathrm{XbaI}$ and EcoRI, and the EcoRI site was filled in by treatment with the Klenow fragment of DNA polymerase I. The 
intron fragment was then ligated to 35S-RVP, which had been linearized with ClaI, blunt-ended with Klenow, and digested with Xbal.

To construct Cl-Sh-GUS, a 5 '-flanking fragment spanning bases -836 to +20 , relative to the transcription start of the $C 1$ gene (Paz-Ares et al. 1987), was prepared by PCR amplification from a 9-kb genomic clone of a wild-type $C 1$ allele (Cone et al. 1986). The primers used for the amplification were $5^{\prime}$-CGATCTCGTATGATGAACC- $3^{\prime}$ (sense strand) and 5'-CATCGCGCTAGCTCTCTCGT-3' (antisense strand). The $3^{\prime}$ primer included a single-base change at +11 to create a unique NheI site. The amplified product was cut with $E c o$ RI and NheI to generate a fragment that spanned -609 to +10 . This fragment was cloned into EcoRI and SpeI-digested pBluescript| - | (Stratagene, Inc.). A HindIII-XbaI fragment containing the $C 1$ promoter was removed from this plasmid and used to replace the CaMV 35S promoter sequence in a derivative of pBI22I (Bevan 1984) in which an EcoRI site at the $3^{\prime}$ end of the NOS terminator had been eliminated. The resulting plasmid was designated $\mathrm{Cl}$ GUS. An $X b a I-E c o R V$ fragment containing the Shl first intron was prepared from the Bluescript subclone described above and ligated to Cl-GUS, which had been digested with $\mathrm{XbaI}$ and $S m a I$. This placed the Shl intron between the $C 1$ promoter and the GUS-coding sequence. A slightly altered version, 35S-ShGUS(d00), was constructed using a Shl intron fragment prepared by digesting the pBluescript Shl subclone with EcoRI, blunting the ends with the Klenow fragment, digesting this fragment with $X b a I$, and inserting the intron fragment into the $X b a I$ and SmaI site of Cl-GUS.

Plasmid 35S-Sh-GUS(d00) was used to construct a series of 5 -deletion derivatives, $\mathrm{d} 10, \mathrm{~d} 20$, and $\mathrm{d} 30$, with end points at $-327,-235$, and -130 , respectively, using NdeI, Sau3A, and SphI sites located at these positions. To make d20, the Sau3A fragment between -235 and -78 was first cloned into the BamHI site of pUC19 so that position -235 was proximal to the EcoRI site of the vector. An EcoRI-SphI fragment of this plasmid (spanning -235 to -135 of the $C 1$ sequence) was ligated to the EcoRI-SphI backbone of 35S-Sh-GUS(d00). By this manipulation, SstI, KpnI, and SmaI sites of the pUC19 polylinker were inserted between the EcoRI site and the 5' end of the $C 1$ sequence in the $\mathrm{d} 20$ derivative. The $\mathrm{d} 10$ derivative was obtained by replacing the sequence between $S m a I$ and $X b a I$ of $\mathrm{d} 20$ with the StuI $(-334) / X b a I$ fragment after end-filling the StuI overhang by treatment with the Klenow fragment of DNA polymerase I. The $\mathrm{d} 30$ deletion was made by digesting 35S-Sh-GUS with EcoRI and SphI, treating with T4 DNA polymerase, and circularizing with T4 DNA ligase. The d29 deletion construct with end point at -157 was generated from d 20 by replacing the SmaI-SphI fragment with a double-stranded synthetic oligonucleotide. The synthetic oligonucleotides were prepared as follows: Oligonucleotide 5'-CATGACAGCAGTGTCGTGTCGTCCATCATGCACTTTAGG-3' was phosphorylated with T4 polynucleotide kinase and annealed with the complementary oligonucleotide 5'-CATGCCTAAAGTGCATGCATGGACGACACGACACTGCTGTA-3'. After filling in the 4-base overhangs with Klenow fragment, the double-stranded oligonucleotide was cut with $S p h \mathrm{I}$ and ligated with the $S m a \mathrm{I} / S p h \mathrm{I}$-digested d 20 vector.

Internal deletion constructs Id $2, \mathrm{Id} 3$, and Id 23 were derivatives of $\mathrm{d} 20$. The $0.6-\mathrm{kb}$ EcoRI-Xbal fragment of C1-Sh-GUS containing the $C 1$ promoter was cloned into pSLECT-1 (Promega), and site-directed mutagenesis was carried out using the Altersite kit (Promega) according to the manufacturer's instructions. The mutagenic primers used to make the precursors of Id 2 and Id 3 were $5^{\prime}-{ }^{107}$ TCAACTCGGCCAACTAGTTAGCGCCA-3' and $5^{\prime}{ }^{-192}$ TTAACTGCGTACGTTGCCC-3', respec- tively. These primers created unique SpeI and SplI sites at positions -95 and -184 , respectively. The pSLECT-1 subclone with a new SpeI site was digested with SpeI, treated with S1 nuclease, and circularized with T4 ligase. By sequencing of the recovered plasmid, we found a 12 -bp deletion from -99 to -88 rather than the expected 4-bp deletion. The mutated fragment was recovered by digestion with $S p h \mathrm{I}$ and $\mathrm{XbaI}$ and ligated to the SphI-XbaI backbone of d20 to generate Id2. Similar treatment of the plasmid with a new SplI site resulted in a 9-bp deletion from -184 to -177 . The Sau3A-SphI fragment of this plasmid, which spans from -235 to -135 relative to wild type, was first subcloned into the BamHI-SphI site of pUC19 and then isolated as an EcoRI-SphI fragment; after ligation to the EcoRI-Sphl backbone of d20, this generated Id3. The same EcoRI-SphI fragment was ligated to the EcoRI-SphI backbone of Id 2 to generate the double mutant Id23. Id4 (a plasmid facsimile of the $c 1-p$ deletion) was made by replacing the sequence between the SmaI and SphI sites of d20 with a synthetic double-stranded oligonucleotide corresponding to a 5-bp deletion of -151 to -147 . The oligonucleotides were prepared as follows: Partially complementary, synthetic oligonucleotides 5'-TA CCCGGGGATCAGTTTTCGTTCGTAGTCAGTTTTCGATAAATGCCAATTTTTTAACTGCATACGTTGCCCTTG-3' (74-mer) and 5'-AAAGTGCATGCATGGACGACACTGCTGTGCTGGTGCTGAGCA AGGGCAACGTATGCAGTT-3' (60-mer) were anealed, treated with the Klenow fragment of DNA polymerase I to complete both strands, and digested with SmaI and SphI. The DNA fragment was purified by polyacrylamide gel electrophoresis prior to ligation. Id5 was obtained by digesting $\mathrm{d} 20$ with $S p h \mathrm{I}$, treating with $\mathrm{Sl}$ nuclease, and circularizing with $\mathrm{T} 4$ ligase. This resulted in a 6-bp deletion from -136 to -131 , confirmed by DNA sequencing.

To construct multiple-base-change mutants 7677, 7879, 8081 , and 8283 , a Stul site was introduced at position -117 in d20 to create d20(Stu). This was accomplished by PCR amplification of a fragment spanning -137 to +10 of the $C 1$ sequence using a $5^{\prime}$ primer that spanned the SphI site $(-135)$ and that incorporated a single $\mathrm{G} \rightarrow \mathrm{A}$ base change at -117 . The amplified fragment was digested with SphI and SalI, and the resulting gel-purified SphI-Sall fragment was ligated to a backbone fragment obtained by SphI and SalI digestion of d20. The 7677, 7879,8081 , and 8283 derivatives were obtained by digesting d20 $\{\mathrm{Stu}\}$ with SphI and StuI and then ligating in double-stranded synthetic oligonucleotide fragments that included SphI compatible and blunt ends. The complementary pairs of oligonucleotides were as follows: For 7677, 5'-CACTTTAGGTGCAGTT-3' and 5'-AACTGCACCTAAAGTGCATG-3'; for 7879, 5'-CACTTTAGTGCTCGGG-3' and 5'-CCCGAGCACTAAAGTGCATG-3'; for 8081, 5'-CACTGCTCGTGCAGGG-3' and 5'-CCCTGCACGAGCAGTGCATG-3'; for 8283, 5' -GCTCTTAGGTGCAGGG-3' and 5'-CCCTGCACCTAAGAGCCATG$3^{\prime}$. The longer oligonucleotide of each pair was phosphorylated with $\mathrm{T} 4$ polynucleotide kinase prior to annealing and ligation. To construct Id6 (-114 to -58$)$, d20(Stu) was digested with StuI and NaeI and recircularized with T4 ligase.

\section{Acknowledgments}

We thank Brian Scheffler, Udo Wienand, and Hienz Seadler for sharing data on the structure of $c 1-p$ prior to publication. This work was supported by grants to D.R.M. from the National Science Foundation (DCB-87-18374) and the McKnight Foundation and to I.K.V. by Monsanto Corporation (St. Louis, MO). This paper is Florida Agriculture Experiment Station Journal series no. R-02249. 
The publication costs of this article were defrayed in part by payment of page charges. This article must therefore be hereby marked "advertisement" in accordance with 18 USC section 1734 solely to indicate this fact.

\section{References}

Bevan, M. 1984. Binary Agrobacterium vectors for plant transformation. Nucleic Acids Res. 12: 8711.

Bradford, M.M. 1976. A rapid and sensitive method for the quantitation of microgram quantities of protein utilizing the principle of protein-dye binding. Anal. Biochem. 72: 248254.

Chen, S.M. and E.H. Coe, Jr. 1978. Control of anthocyanin synthesis by the $C$ locus in maize. Biochem. Genet. 15: 333346.

Coe, E.H. and M.G. Neuffer. 1977. The genetics of corn. In Corn and corn improvement (ed. G.F. Sprague), pp. 111-213. American Society of Agronomy, Madison, WI.

Cone, K.C., F.A. Burr, and B. Burr. 1986. Molecular analysis of the maize anthocyanin regulatory locus c1. Proc. Natl. Acad. Sci. 83: 9631-9635.

Dooner, H.K. 1985. Viviparous-1 mutation in maize conditions pleiotropic enzyme deficiencies in the aleurone. Plant Physiol. 77: 486-488.

Dickinson, C.D., R.P. Evans, and N.C. Neilsen. 1988. RY repeats are conserved in the $5^{\prime}$-flanking regions of legume seed-protein genes. Nucleic Acids Res. 16: 371.

Goff, S., T.M. Klein, B.A. Roth, M.E. Fromm, K.C. Cone, J.P. Radicella, and V. L. Chandler. 1990. Transactivation of anthocyanin biosynthetic genes following transfer of $B$ regulatory genes into maize tissues. EMBO /. 9: 2517-2522.

Goff, S., K.C. Cone, and M.E. Fromm. 1991. Identification of functional domains in the maize transcriptional activator $\mathrm{Cl}$ : Comparison of wild-type and dominant inhibitor proteins. Genes \& Dev. 5: 298-309.

Guiltinan, M.J., W.R. Marcotte, and R.S. Quatrano. 1990. A lcucine zipper protein that recognizes an abscisic acid response element. Science 250: 267-270.

Jefferson, R.A. 1987. Assaying chimeric genes in plants: The GUS gene fusion system. Plant Mol. Biol. Rep. 5: 387-405.

Jones, R.J. and M.L. Brenner. 1987. Distribution of abscisic acid in maize kernel during grain filling. Plant Physiol. 83: 905909.

Klein, T.M., T. Gradziel, M.E. Fromm, and J.C. Sanford. 1988. Factors influencing gene delivery into Zea mays cells by high-velocity microprojectiles. Biotechnology 6: 559-563.

Ludwig, S.R., L.F. Habera, S.L. Delaporta, and S.R. Wessler. 1989. $L c$, a member of the maize $R$ gene family responsible for tissue-specific anthocyanin production, encodes protein similar to transcriptional activators and contains the mychomology region. Proc. Natl. Acad. Sci. 86: 7092-7096.

Maniatis, T., E.F. Fritsch, and J. Sambrook. 1982. Molecular cloning: A laboratory manual. Cold Spring Harbor Laboratory, Cold Spring Harbor, New York.

Marcotte, W.R., Jr., C.C. Bayley, and R.S. Quatrano. 1988. Regulation of a wheat promoter by abscisic acid in rice protoplasts. Nature 335: 454-457.

Marcotte, W.R. Jr., S.H. Russell, and R.S. Quatrano. 1989. Abscisic acid response sequences from the Em gene of wheat. Plant Cell 1: 969-976.

McCarty, D.R. and C.B. Carson. 1990. The molecular genetics of seed maturation in maize. Physiol. Plant. 81: 267-272.

McCarty, D.R., C.B. Carson, M. Lazar, and S.C. Simonds. 1989a. Transposable element induced mutations of the vivipa- rous-1 gene of maize. Dev. Genet. 10: 473-481.

McCarty, D.R., C.B. Carson, P.S. Stinard, and D.S. Robertson. 1989b. Molecular analysis of viviparous-1: An abscisic acid insensitive mutant of maize. Plant Cell 1: 523-532.

McCarty, D.R., T. Hattori, C.B. Carson, V. Vasil, and I.K. Vasil. 1991. The viviparous-1 developmental gene of maize encodes a novel transcriptional activator. Cell 66: 895-905.

Mundy, J., K. Yamaguchi-Shinozaki, and N.-H. Chua. 1990. Nuclear proteins bind conserved elements in the abscisic acid responsive promoter of a rice rab gene. Proc. Natl. Acad. Sci. 87: 406-410.

Murashige, T. and F. Skoog. 1962. A revised medium for rapid growth and bioassays with tobacco tissue cultures. Physiol. Plant. 15: 473-497.

Neill, S.J., R. Horgan, and A.D. Parry. 1986. The carotenoid and abscisic acid content of viviparous kernels and seedlings of Zea mays L. Planta 169: 87-96.

Paz-Ares, J., D. Ghosal, U. Wienand, P. Peterson, and H. Saedler. 1987. The regulatory locus $c 1$ of Zea mays encodes a protein with homology to myb proto-oncogene products and with structural similarities to transcriptional activators. EMBO $/$. 6: $3553-3558$

Robertson, D.S. 1955. The genetics of vivipary in maize. Genetics 40: 745-760.

Robichaud, C.S. and I.M. Sussex. 1986. The response of viviparous-1 and wildtype embryos of Zea mays to culture in the presence of abscisic acid. 1. Plant Physiol. 126: 235-242.

1987. The uptake and metabolism of ${ }^{14} \mathrm{C}-\mathrm{ABA}$ by excised wildtype and viviparous-1 embryos of Zea-mays L. $/$. Plant Physiol. 130: 181-188.

Robichaud, C.S., J. Wong, and I.M. Sussex. 1980. Control of in vitro growth of viviparous embryo mutants of maize by abscisic acid. Dev. Genet. 1: 325-330.

Roth, B.A., S.A. Goff, T.M. Klein, and M.E. Fromm. 1991. C1 and $R 1$ dependent expression of the maize $B z 1$ gene requires sequences with homology to mammalian myb and myc binding sites. Plant Cell 3: 317-325.

Skriver, K. and J. Mundy. 1990. Gene expression in response to abscisic acid and osmotic stress. Plant Cell 2: 503-512.

Triezenberg, S.J., K.L. Marco, and S.L. McKnight. 1988. Evidence of DNA: Protein interactions that mediate HSV-1 immediate early gene activation by VP16. Genes \& Dev. 2: $730-742$.

Vasil, V., M. Clancy, R.J. Ferl, I.K. Vasil, and L.C. Hannah. 1989. Increased gene expression by the first intron of the maize $s h 1$ locus in grass species. Plant Physiol. 91: 1575-1579.

Vilardell, J., A. Goday, M.A. Freire, M. Torrent, M.C. Martinez, J.M. Torne, and M. Pages. 1990. Gene sequence, developmental expression and protein phosphorylation of RAB17 in maize. Plant Mol. Biol. 14: 423-432.

Wienand, U., J. Paz-Ares, B. Scheffler, and H. Saedler. 1990. Molecular analysis of gene regulation in the anthocyanin pathway of Zea mays. In Plant gene transfer (ed. C.J. Lamb and R.N. Beachyl, pp. 111-124. Wiley Press, New York.

Zack, C.D., R.J. Ferl, and L.C. Hannah. 1986. DNA sequence of a shrunken allele of maize: evidence for visitation by insertional sequences. Maydica 31: 5-16.

Zenke, M., A. Wilderman, and P. Chambon. 1987. The SV40 early promoter. In Molecular aspects of papovaviruses (ed. Y. Aloni), pp. 85-100. Martinus Nijhoff, Boston, MA. 


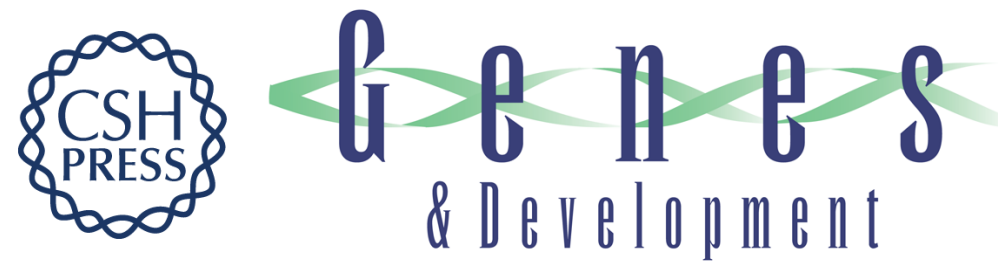

\section{The Viviparous-1 gene and abscisic acid activate the $C 1$ regulatory gene for anthocyanin biosynthesis during seed maturation in maize.}

T Hattori, V Vasil, L Rosenkrans, et al.

Genes Dev. 1992, 6:

Access the most recent version at doi:10.1101/gad.6.4.609

References This article cites 33 articles, 13 of which can be accessed free at:

http://genesdev.cshlp.org/content/6/4/609.full.html\#ref-list-1

License

Email Alerting

Service right corner of the article or click here.

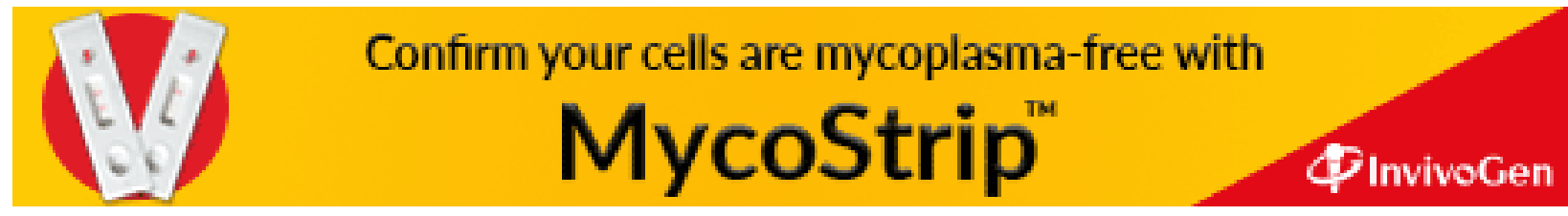

